
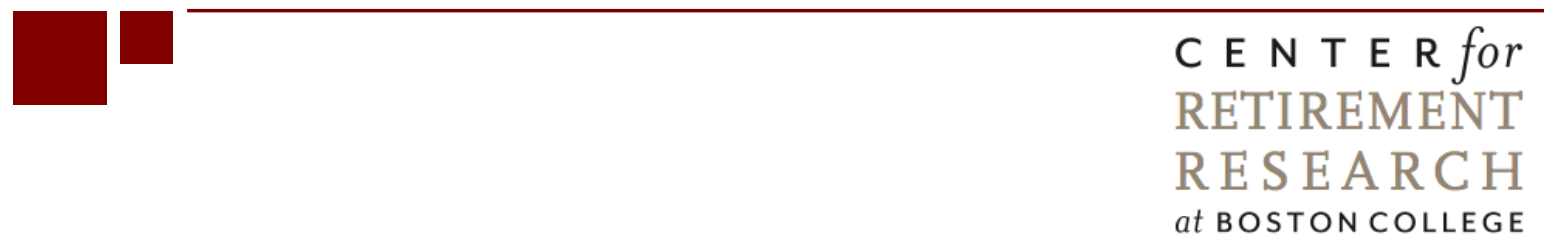

\title{
TRENDS IN OPIOID USE AMONG SOCIAL SECURITY DISABILITY INSURANCE APPLICANTS
}

\author{
April Yanyuan Wu, Denise Hoffman, and Paul O’Leary \\ CRR WP 2021-6 \\ March 2021 \\ Center for Retirement Research at Boston College \\ Hovey House \\ 140 Commonwealth Avenue \\ Chestnut Hill, MA 02467
}

Tel: 617-552-1762 Fax: 617-552-0191

https://crr.bc.edu

\begin{abstract}
April Yanyuan Wu is a researcher at the Mathematica Center for Studying Disability Policy. Denise Hoffman is a senior researcher at the Mathematica Center for Studying Disability Policy. Paul O'Leary is an economist at the U.S. Social Security Administration. The research reported herein was derived in whole or in part from research activities performed pursuant to a grant from the U.S. Social Security Administration (SSA) funded as part of the Retirement and Disability Research Consortium. The opinions and conclusions expressed are solely those of the authors and do not represent the opinions or policy of SSA, any agency of the federal government, Mathematica - Center for Studying Disability Policy, or Boston College. Neither the United States Government nor any agency thereof, nor any of their employees, make any warranty, express or implied, or assumes any legal liability or responsibility for the accuracy, completeness, or usefulness of the contents of this report. Reference herein to any specific commercial product, process or service by trade name, trademark, manufacturer, or otherwise does not necessarily constitute or imply endorsement, recommendation or favoring by the United States Government or any agency thereof. The authors wish to thank David Stapleton for his insightful comments on the draft manuscript. They are also grateful to Peter Mariani, Sooin Lee, and Serge Lukashanets of Mathematica for their help with the machine-learning method and the interpretation of the data used in this analysis. In addition, the authors acknowledge Olivier Bodenreider of the National Library of Medicine, National Institutes of Health, for his help with RxNorm.
\end{abstract}

(C) 2021, April Yanyuan Wu, Denise Hoffman, and Paul O'Leary. All rights reserved. Short sections of text, not to exceed two paragraphs, may be quoted without explicit permission provided that full credit, including $(\mathrm{C}$ notice, is given to the source. 


\begin{abstract}
About the Center for Retirement Research
The Center for Retirement Research at Boston College, part of a consortium that includes parallel centers at the National Bureau of Economic Research, the University of Michigan, and the University of Wisconsin-Madison, was established in 1998 through a grant from the U.S. Social Security Administration. The Center's mission is to produce first-class research and forge a strong link between the academic community and decision-makers in the public and private sectors around an issue of critical importance to the nation's future. To achieve this mission, the Center conducts a wide variety of research projects, transmits new findings to a broad audience, trains new scholars, and broadens access to valuable data sources.
\end{abstract}

Center for Retirement Research at Boston College

Hovey House

140 Commonwealth Ave

Chestnut Hill, MA 02467

Tel: 617-552-1762 Fax: 617-552-0191

https://crr.bc.edu

Affiliated Institutions:

The Brookings Institution

Mathematica - Center for Studying Disability Policy

Syracuse University

Urban Institute 


\begin{abstract}
Several factors suggest that opioid use may be common among applicants to Social Security Disability Insurance (SSDI): the prevalence of opioid use, the suspected link between opioid use and declining rates of work, and the large share of new SSDI awardees who have conditions associated with opioid use. However, research-ready data on opioid use by these applicants, who are generally not eligible for Medicare, have not been available. SSDI applicants are required to report their medications, but they often do so in an open-ended text field, which requires additional coding before analysis.

Our study is the first to provide statistics on opioid use among SSDI applicants. We used an innovative machine-learning method to identify opioids in medication text fields in SSDI administrative data. Specifically, we examined the prevalence of reported opioid use in a 30 percent random sample of initial-level SSDI applications stored in the Social Security Administration's Structured Data Repository (SDR) from 2007 through 2017, considering differences by demographic and other factors. We supplemented the SDR with two SSA administrative data sources: the Disability Analysis File, which provided award information, and the Numerical Identification System, which provided information on deaths. Using these sources, we produced statistics on the association between (1) opioid use among SSDI applicants and (2) SSDI award and death. Understanding the prevalence of reported opioid use among these individuals and the association between opioid use and later SSDI application outcomes may help in forecasting the future composition of the SSDI caseload.
\end{abstract}

The paper found that:

- Over the 11-year analysis period, more than 30 percent of SSDI applicants reported using one or more opioids. This is higher than the rate of opioid use in the general population (29 versus 19 percent in 2016).

- Reported rates of opioid use among SSDI applicants varied over the analysis period. Rates increased from 2007 to a peak of 32 percent in 2012, followed by a decline to the period low of 26 percent in 2017.

- Reported opioid use varied by age and demographic characteristics. SSDI applicants ages 40-49 were the most likely age group to report opioid use; women were 3-4 
percentage points more likely to report opioid use than men; and people with some college were the most likely education group to report opioid use.

- Reported opioid use is also correlated with application type. SSDI-only applicants who reported opioid use were 4-6 percentage points more likely to report opioid use than concurrent SSDI and SSI applicants.

- Reported opioid use varied greatly between geographic areas. Applicants from Rhode Island, Massachusetts, and Washington, DC, reported lower-than-average rates of opioid use in 2007 and consistently throughout the analysis period. Conversely, applicants from Delaware, Nevada, and Michigan consistently reported the highest rates of opioid use.

- There was a positive and statistically significant association between (1) reported opioid use and SSDI awards and (2) reported opioid use SSDI award and death. These associations do not demonstrate a causal relationship.

The policy implications of the findings are:

- Application for SSDI provides an opportunity to identify opioid users, understand more about the nature of their use, and, if warranted, connect them with helpful services and supports.

- Given the prevalence of reported opioid use among SSDI applicants, our study may open the door to future research on how opioid use affects post-adjudication well-being-for example, applicants' employment outcomes after they are awarded or denied SSDI.

Future studies might also consider tracking the trajectory of opioid use from application through award using Medicare data.

- This study also serves as a template for using previously untapped information in SSA administrative files. The machine learning approach used to identify opioid use in freeform text could be applied to other key indicators of interest to SSA. 


\section{Introduction}

Social Security Disability Insurance (SSDI) is the nation's largest safety net program for former workers with disabilities and their dependents, providing cash benefits to over 10 million beneficiaries in 2018 (Social Security Administration [SSA] 2018). To be eligible, workers must have a long-lasting physical or mental impairment that precludes substantial employment, among other requirements. SSDI applicants may qualify for benefits based on a wide variety of impairments - although, notably, they may not qualify solely based on drug addiction or if addiction is a contributing factor material to the determination. Regardless, SSDI applicants and beneficiaries may use prescription drugs, such as opioids, with varying outcomes ranging from successful therapeutic use to misuse and addiction.

The United States has seen a surge in opioid use over the past two decades. According to the U.S. Department of Health and Human Services (2019), the opioid epidemic began in the late 1990s, when health care providers began to prescribe opioids at greater rates, reassured by pharmaceutical companies that patients would not become addicted. Opioids initially made headway in the United States as a treatment for cancer pain and then began to be used to treat non-cancer pain more broadly in the mid- to late-1990s (Jones et al. 2018). Nationwide, prescription rates peaked in 2012, with 81 opioid prescriptions written for every 100 Americans (Centers for Disease Control and Prevention [CDC] 2019a).

During that time, it became clear that opioids can be highly addictive: 21 to 29 percent of patients prescribed opioids for pain misused them (Vowles et al. 2015). Opioids were also responsible for more than 700,000 overdose deaths from 1999 to 2017 (CDC 2019b). Although opioid use has recently declined, in 2017 there were still 59 opioid prescriptions written for every 100 Americans (CDC 2018a); in 201619 percent of the U.S. population filled at least one opioid prescription (Mytelka et al. 2018).

The impact of the rise in opioid use could have disparate effects on health and work capacity. On one hand, opioids may ease users' pain, which could help them engage in work and other activities. This appears to be the case with non-opioid pain relievers, which have been found to increase labor force participation and lower absenteeism (Garthwaite 2012; Bütikofer and Skira 2018).

However, opioid use comes with a high risk of dependency that may exacerbate other health conditions, and could interfere with work. For instance, Scherrer et al. (2016) found that 
prescription opioids are associated with an increased risk of depression. In another study, Schiltenwolf et al. (2014) found that opioids may further impair cognition and executive function in elderly and other patients already at risk of such impairments. Furthermore, a recent paper by Harris et al. (2019) shows that a 10 percent increase in opioid prescriptions reduces labor force participation by more than 0.5 percentage points.

The prevalence of opioid use nationwide, the suspected link between opioid use and declining work, and the large share of new SSDI awardees who have conditions associated with opioid use suggest that opioid use may be common among SSDI applicants. From 2007 to 2016, there was a rise in SSDI awards made to applicants with musculoskeletal conditions (SSA 2019), which are often associated with opioid use (Carnide et al. 2017). Furthermore, a recent analysis by Cutler et al. (2017) suggests that the wider availability of opioids is linked to increases in SSDI applications. In addition, 44 percent of SSDI awardees who have completed the two-year waiting period for Medicare filled at least one opioid prescription in 2011 (Morden et al. 2014).

Statistics on opioid use among SSDI applicants, who are generally not eligible for Medicare, have been unavailable because of the lack of research-ready data. SSDI applicants are required to report their medications, but they often do so in an open-ended text field. In the past, analyzing this information required manual coding, a process that is labor intensive, time consuming, and usually cost prohibitive.

Our study is the first to provide statistics on opioid use among SSDI applicants. We used an innovative machine-learning method to identify opioids in medication text fields in SSDI administrative data. Specifically, we documented the overall prevalence of opioid use among SSDI applicants from 2007 through 2017, considering differences by demographic and other factors. We also produced statistics on the association between (1) opioid use among SSDI applicants and (2) SSDI award and death. Understanding the prevalence of opioid use among SSDI applicants and the association between opioid use and later SSDI application outcomes may help stakeholders forecast the future composition of the SSDI caseload.

\section{Data}

We used data from SSA's Structured Data Repository (SDR) for our analysis. Since 2007, SSA has electronically stored data from SSDI and Supplemental Security Income (SSI) 
applications in the SDR ${ }^{1}$. The application form asks "Are you taking any medicines (prescription or non-prescription)?" and, if the respondent answers affirmatively, he or she is asked to list the name of the medicine. Applicants who complete an electronic version of the application may report medications using a pull-down list of 630 medication names, enter their medications in a free-text field, or both. Applicants who complete a paper application must list their medications in a free-text field.

Our analysis is based on a 30 percent random sample of initial-level SSDI applications stored in the SDR each year from 2007 through 2017. We used the effective filing date as the basis for the application year. We retained the first application per person per year and included all cases with award or denial decisions at the initial level over this period where the applicant was age 18 to 67 at the time of application, they applied for SSDI, either alone or together with SSI benefits, and had a valid decision code. The sample included both SSDI-only and concurrent SSDI and SSI applications. The sample excludes SSI-only applications. In total, the sample consisted of 5,954,422 applications and nearly 26 million reported medications.

Applicants in our sample mostly reported medications through free-text entries (Exhibit 1). During our analysis period, 45 percent of applicants who reported medications did so exclusively in free text, and another 39 percent used free text along with the pull-down list. This highlights the importance of free-text entries in analyzing medication information. The challenge with this type of analysis is that free-text data are not standard and may include nonmedication names, extraneous information such as dosage, or misspellings.

We supplemented the SDR with two other SSA administrative data sources: we used the Disability Analysis File to obtain award information and the Numerical Identification System (Numident) for information on death.

It is important to note that this analysis is based on self-reported use of medicine. If applicants underreport opioids, any estimates based on these data will underestimate the true rates of opioid use among this population. In addition, although the application form instructs applicants to report prescription and non-prescription medication, it seems likely that use of nonprescription opioids will be underreported. It is also possible that accurate opioid reporting varies

\footnotetext{
${ }^{1}$ Note that, although the SDR is thought to include the large majority of SSDI applications, it does not include all of them. Specifically, cases that do not undergo the standard disability determination process are not included in the SDR. These may include second (and higher order) applications in which the new application provides no new information from the previous application, as well as technical denials, such as cases in which the claimant dies before adjudication begins or the claimant does not wish to pursue the claim.
} 
by subgroup, meaning that opioid use could be underreported to different extents for different applicant subgroups.

\section{Methods}

\section{Identifying Opioids}

To identify opioids in the free-text entries, we used a supervised machine-learning algorithm based on natural-language processing (NLP). This process involved several steps. First, we extracted a random subset of 2,500 unique free-text medication entries. Given the repeating pattern of the data, this subset represents over 30 percent of all unique medication values in the sample. A subject matter expert manually labeled each entry as an opioid or nonopioid medication. We then divided the labeled data into a training set and a testing set in a ratio of 3:1. We supplemented the training data with a sample of about 1,000 labeled free-text medication entries from 2013, extracted when originally developing this machine-learning algorithm as a proof of concept (Wu et al. 2019).

Using the training set, we created an algorithm in Python to identify opioids. We then separated the free-text entries into tokens, which are either single words or multiple words that have special meaning when grouped. We referred to an opioids list maintained by the CDC to identify multiword tokens. Next, we removed any tokens unlikely to be drugs; these were tokens that started with a non-letter value; English language words that appeared in the Words Corpus dictionary in the Python NLP library; and common abbreviations such as "mgs" and "tabs."

Our next step was to develop an algorithm to compare the remaining words (which we suspected to be medication names) to lists of medications, including CDC's opioid list and the National Library of Medicine's RxNorm resource of opioid and non-opioid drug names. We compared free-text entries to CDC's opioid list using the Damerau-Levenshtein distance and the phonetic distance from the true spelling of the opioid name. The Damerau-Levenshtein distance represents the number of insertions, deletions, and substitutions required to change one word to another. Phonetic distance measures the distance between phonetic encodings of the opioid and of the free-text entries. We also compared entries to exact matches or RxNorm-suggested approximate matches to opioids and non-opioids.

For the model development, we compared the list of opioids and non-opioids classified by the subject matter expert to the above indicators, including (1) whether a medication appears 
in the dictionary, (2) the Damerau-Levenshtein distance of a medication to a CDC opioid name, (3) the phonetic distance of a medication to a CDC opioid name, (4) whether a medication exact matches with an RxNorm opioid name or suggested approximate matches, and (5) whether a medication exact matches with a non-opioid name in RxNorm or suggested approximate matches. We tested numerous models to identify the most effective combination and specification of these five NLP-based classifications and determined that a logistic regression classification was best. This process also provided additional information, such as the best Damerau-Levenshtein distance to a CDC opioid, which was 0 or 1.

After developing the algorithm, we applied it to the testing set data-which was not used for training - to assess its performance. We achieved an accuracy rate of 99.9 percent, with a sensitivity of 99.2 percent and specificity of 100 percent. This means that our algorithm made very few mistakes on the free-text cases in the testing sample, and the kinds of mistakes it made were undercounting a small proportion of true opioid cases.

After testing, we used the machine learning algorithm to code the remaining unlabeled text records. We used a similar approach to classify medications from the pull-down list. Drawing from both modes of medication reporting, we created application-level indicators for any opioid use and number of reported opioids.

\section{Analyzing Data on Opioid Use}

We drew on information in the SDR to describe rates of reported opioid use over time for all applicants and across applicant subgroups. Specifically, we compared rates of reported opioid use among applicants by age, gender, education, concurrent application for SSI, and state of residence. We do not produce crosstabs for opioids and other characteristics because the SDR does not have research-ready data on other variables of interest. Much of the application data are entered and stored as free-field text, which is resource intensive to transform into data that can be used in a quantitative analysis.

To explore the extent to which reported opioid use correlates to later outcomes, we followed the 30 percent random sample of the cohort of 2009 applicants and examined their outcomes in 2014. Specifically, we identified whether each applicant was awarded benefits through the end of 2014 and whether he or she had died as of the start of 2014. Our information 
on benefit award came from the Disability Analysis File, and information on death came from the Numident file.

We estimated a multinomial logit model with four outcomes: (1) awarded SSDI benefits and alive in 2014, (2) awarded SSDI benefits and dead by 2014, (3) not awarded SSDI benefits and alive in 2014, and (4) not awarded SSDI benefits and dead by 2014. We controlled for beneficiary characteristics such as age, gender, education level, geographic region, past or scheduled medical care for a physical condition, past or scheduled medical care for a mental condition, and concurrent application to SSI.

\section{Results}

During our analysis period (2007 to 2017), one-quarter to one-third of SSDI applicants reported any opioid use (Exhibit 2). In 2007, 28 percent of applicants reported using opioidsthat rose to a peak of 32 percent in 2012 before dropping to a low of 26 percent in 2017. This mirrors nationwide trends, which show a rise in opioid prescribing from 2007 to 2012 followed by a decline from 2013 to 2017 (CDC 2018a).

Over our 11-year analysis period, 1,793,414 applications in our 30 percent analysis sample indicated opioid use. These applications may represent up to 6 million applicants, although it is possible that the same people may have submitted several applications over time. Nonetheless, this highlights SSDI application as an important point of contact between opioid users and a federal program.

\section{Rate of Opioid Use by Beneficiary Characteristics}

Reported opioid use varied by the demographic characteristics of SSDI applicants, such as their gender. Throughout the analysis period, the share of women who reported opioid use was 11 to 15 percent higher than the share of men - a statistically significant difference (Exhibit 3). For example, 34 percent of female applicants reported any opioid use in 2012 versus 30 percent of male applicants. This is in line with nationwide trends indicating more use of prescription opioids among women than among men (Serdarevic et al. 2017; CDC 2018b). 
Reported opioid use also varied notably by age (Exhibit 3). Reported use was highest among applicants ages 40 to 49 (31 to 37 percent) and was roughly half as prevalent among applicants ages 18 to 29 (13 to 18 percent). Older age groups were less likely to report opioid use than those ages 40 to 49: 29 to 35 percent of those ages 50 to 59 reported opioid use, as did 24 to 29 percent of those ages 60 to 67 . This pattern deviates from nationwide trends, which show opioid use rising with age (CDC 2018b).

We also saw variation in reported opioid use by education (Exhibit 3). We present results only for sample members who are ages 22 and above to account for the possibility that younger applicants are most likely to complete additional years of education (which would not be captured by the data). ${ }^{2}$ We found that reported opioid use was highest among applicants with some postsecondary education, followed by those with a high school degree or GED and those with a college degree. Reported use was lowest among applicants with less than a high school degree. These results are somewhat surprising given that higher rates of opioid prescribing are associated with lower educational attainment, holding other characteristics constant (CDC 2017). It is possible that our results reflect an association between low education and other characteristics that drive the observed relationship.

Reported opioid use was also correlated with application type (Exhibit 3). The proportion of SSDI-only applicants who reported opioid use was 15-25 percent higher than the proportion of opioid users who concurrently applied to SSDI and SSI. The differences, ranging from 4-6 percentage points, were statistically significant. This finding could be related to different sources of health insurance. In the 1990s, although awarded DI applicants and awarded SSI applicants had similar rates of insurance coverage at the time of application, but DI applicants had higher rates of employer-sponsored insurance (61 percent versus 29 percent) (Gruber and Kubik 2002). Of course, the health insurance landscape has changed dramatically since this study was conducted and it's possible these relationships have changed.

Finally, we saw variation in reported opioid use across states (Exhibit 4). Among all states collectively, we saw the same overall pattern as for the full sample: a rise in reported opioid use leading up to and including 2012, followed by a decline. Applicants from Washington, DC; Rhode Island; and Massachusetts consistently reported the lowest rates of use in the country, whereas applicants from Delaware, Nevada, and Michigan consistently reported

2 The results are generally the same when we include applicants under age 22. 
the highest rates. In the state with the lowest rate, Washington, DC, less than 16 percent of applicants reported opioid use in 2017. In the state with the highest rate, Delaware, more than 41 percent of applicants reported opioid use in 2012. We also observed several deviations from general trends in states. For example, Arkansas saw an increase in reported opioid use from more than 27 percent in 2007 to 32 percent in 2017.

The state-level rates of reported opioid use among SSDI applicants did not always correspond to the statewide rates of opioid prescribing. In 2017, for example, applicants from Maine had the 3rd lowest rate of reported opioid use, but Maine also had the 21st lowest rate of opioid prescriptions (CDC 2018b). Applicants from Mississippi had the 27th highest rate of reported opioid use, but Mississippi also had the 4th highest rate of opioid prescriptions. There was, however, some alignment between reported opioid use and opioid prescribing. For example, Washington, DC, had the lowest opioid prescription rate in 2017, and applicants from DC also reported the lowest rates of opioid use that year.

\section{Association Between Opioid Use and Later Outcomes}

We used a multinomial logit model to examine the association between reported opioid use at SSDI application and subsequent outcomes. Among 2009 applicants, opioid use reported at the time of application was associated with several outcomes five years later (Exhibit 5). Applicants who reported opioid use were 4.1 percentage points more likely to be awarded SSDI benefits and to be alive through the end of 2014. This represents an 8 percent increase over the 51.6 percent of applicants who did not report using opioids. Applicants who reported opioid use were also 1.5 percentage points more likely to be awarded SSDI benefits and to have died by 2014 - a 26 percent increase relative to those not reporting opioid use, although this is from a small base.

Reported opioid use was also associated with a 0.8 percentage point increase in the chance of being denied SSDI benefits and deceased by 2014, a 25 percent increase over the group that did not report opioid use. In addition, applicants who reported opioid use were 6.5 percentage points less likely to have been denied SSDI benefits and to be alive in 2014, a 16 percent decrease over those not reporting opioid use. All differences are statistically significant at the 1 percent level. 
Collectively, the multinomial results suggest a positive and statistically significant association between (1) reported opioid use and (2) SSDI awards and deaths. Both SSDI awards and deaths indicate worse health conditions than those of people who were denied SSDI benefits and who lived more than five years after application. Hence, reported opioid use may also signal poor health and severe impairments. However, these observed associations do not necessarily mean that opioid use caused these outcomes; other factors may be important determinants of SSDI award and death, such as a beneficiary's impairment, disability severity, overall health status, and work history. But owing to the lack of research-ready data on applicants in the administrative files, we were unable to control for these other factors.

\section{Discussion}

Our findings confirm that opioid use is common among SSDI applicants. Over the analysis period, over 30 percent of applicants reported using one or more opioids. This is presumably an underestimate of opioid use, assuming applicants underreport opioid use. Nonetheless, the rate of reported opioid use is higher than the rate of opioid use in the general population (29 versus 19 percent in 2016) but lower than the rate among SSDI beneficiaries who have completed the two-year waiting period for Medicare (32 versus 44 percent in 2011; Morden et al. 2014). The lower rate among applicants relative to awardees may reflect that awardees have more severe medical conditions than denied applicants, by program design.

Reported rates of opioid use among SSDI applicants varied over the 11-year analysis period. Opioid use rose from 2007 to its peak in 2012 (32 percent), before declining to the period low in 2017 (26 percent). These trends mirror changes in the rates of opioid prescribing over time, as reported by the CDC. If opioid use among applicants continues to follow trends in prescribing, we would expect to see a continued decline in reported opioid use. However, we cannot be certain whether the changes in applicants' opioid use are driven by prescribing patterns, a change in the composition of applicants, or other factors.

Despite the observed decline in reported opioid use among SSDI applicants, it was still prevalent in 2017. That year, more than one-quarter of all applicants reported opioid use, and these rates were higher among certain applicants. Across all subgroups, the highest rate of reported use was in the 40 to 49 age group: 31 to 37 percent of these applicants used opioids at the time of application. This is notable because, if awarded, these applicants may remain on the 
SSDI rolls for more than 25 years. Furthermore, the results of a multinomial logit analysis suggest a positive association between reported opioid use at the time of application and SSDI award. Although this does not necessarily indicate a causal relationship, it does indicate that a significant share of new SSDI awardees are opioid users.

\section{Limitations}

When interpreting our results, it is important to recognize several limitations to the analysis. First, because the SDR has limited research-ready data on the characteristics of applicants, our estimates of the association between opioid use and subsequent SSDI award and death do not control for several factors that may affect award and death. Notably, we did not include controls for impairment, disability severity, overall health status, and work history.

We also believe our results underestimate the true rates of opioid use among SSDI applicants. The statistics in this paper are based on self-reported opioid use among applicants. Generally, survey respondents tend to underreport stigmatized behaviors and there is a documented tendency of drug users to underreport drug use in surveys, particularly illicit drug use (Center for Behavioral Health Statistics and Quality 2014, Fendrich et al. 2004). Although our data are from administrative rather than survey data, it still seems likely that non-prescription opioid use is underreported, and it is also possible that the degree of underestimation varies by applicant subgroup.

In addition, our analysis focuses on any opioid use, but does not capture nuances related to opioid use. Analysis of the duration of use, opioid potency, and number of opioids used, would paint a more complete picture of opioid use among SSDI applicants. But this information is either not available (for example, duration of use) or it is beyond the scope of this paper. It is difficult to measure the number of opioids used because applicants may report the same opioid under several names, including the chemical name and brand name. For example, an applicant could report both use of hydrocodone (a chemical name) and Vicodin (a brand name of a hydrocodone medication).

\section{Conclusion}

This study established that reported opioid use was prevalent among SSDI applicants from 2007 through 2017. Opioid use may affect many facets of the SSDI program, including the 
decision to apply for SSDI, an SSDI award (although opioid use itself cannot warrant an award), and subsequent health outcomes. The positive association between reported opioid use at application and SSDI award suggests that opioid use may be prevalent among new awardees, although it is possible that opioid use may change between application and award. While opioids are an appropriate course of treatment for some applicants, the risk of improper use is significant and the consequences of improper use are severe.

Application for SSDI provides an opportunity to identify opioid users, understand more about the nature of their use, and, if warranted, connect applicants to services and supports. Presumably some applicants use opioids as prescribed and experience the intended therapeutic benefits of the medication. However, this is also an opportunity to identify unhelpful opioid use or opioid abuse and connect those users to services. The reach of this potential intervention point could be notable: during the analysis period, up to 6 million self-reported opioid users applied for SSDI, although presumably opioid use is an appropriate course of action for some users.

Furthermore, given the prevalence of reported opioid use among SSDI applicants, research is needed on how opioids affect their post-adjudication outcomes and well-being. For example, among people who report opioid use when they apply for SSDI, what are their employment outcomes after they are awarded or denied benefits? And how does the trajectory of opioid use changes from application through award. Research on these applicants' capacity for work, work outcomes, health outcomes, reapplication to SSDI, and duration on SSDI will provide more information on this notable group of program applicants. 


\section{References}

Bütikofer, Aline, and Meghan M. Skira. 2018. "Missing Work Is a Pain: The Effect of Cox-2 Inhibitors on Sickness Absence and Disability Pension Receipt." Journal of Human Resources 53(1): 71-122.

Carnide, N., S. Hogg-Johnson, P. Côté, E. Irvin, D. Van Eerd, M. Koehoorn, and A.D. Furlan. 2017. "Early Prescription Opioid Use for Musculoskeletal Disorders and Work Outcomes: A Systematic Review of the Literature." The Clinical Journal of Pain 33(7): 647-658.

Center for Behavioral Health Statistics and Quality. 2014. "National Survey on Drug Use and Health: Summary of Methodological Studies, 1971-2014.” Substance Abuse and Mental Health Services Administration, Rockville, MD.

Centers for Disease Control and Prevention (CDC). 2017. "Vital Signs: Changes in Opioid Prescribing in the United States, 2006-2015." Morbidity and Mortality Weekly Report 66: 697-704. doi: 10.15585/mmwr.mm6626a4.

CDC. 2018a. "Prescription Opioid Data: Prescribing Practices." Atlanta, GA. Available at: https://www.cdc.gov/drugoverdose/data/prescribing.html

CDC. 2018b. "Annual Surveillance Report of Drug-Related Risks and Outcomes, 2018." Atlanta, GA. Available at: https://www.cdc.gov/drugoverdose/pdf/pubs/ 2018-cdc-drug-surveillance-report.pdf

CDC. 2019a. "Prescription Opioid Data: Prescribing Practices - Changes in Opioid-Prescribing Practices." Atlanta, GA. Available at: https://www.cdc.gov/drugoverdose/data/prescribing/prescribing-practices.html

CDC. 2019b. "Opioid Basics: Understanding the Epidemic." Atlanta, GA. Available at: https://www.cdc.gov/drugoverdose/epidemic/index.html

Cutler, David, Ellen Meara, and Susan Stewart. 2017. "Has Wider Availability of Prescription Drugs for Pain Relief Affected SSDI and SSI Enrollment?" Presented at the Disability Research Consortium Annual Meeting (August 2). Washington, DC.

Fendrich, Michael, Timothy P. Johnson, Joseph S. Wislar, Amy Hubbell, Vina Sipehler. 2004. "The Utility of Drug Testing in Epidemiological Research: Results from a General Population Survey.” Addiction 99(2): 197-208.

Jones, Mark R., Omar Viswanath, Jacquelin Peck, Alan D. Kaye, Jatinder S. Gill, Thomas T. Simopoulos. 2018. "A Brief History of the Opioid Epidemic and Strategies for Pain Medicine." Pain and Therapy 7:13-31. doi: 10.1007/s40122-018-0097-6. 
Garthwaite, Craig L. 2012. "The Economic Benefits of Pharmaceutical Innovations: The Case of Cox-2 Inhibitors." American Economic Journal: Applied Economics 4(3): 116-137. doi: 10.1257/app.4.3.116.

Gruber, Jonathan and Jeffrey Kubik. 2002. "Health Insurance Coverage and the Disability Insurance Application Decision.” NBER Working Paper 9148. Cambridge, MA: NBER.

Harris, Matthew C., Lawrence M. Kessler, Matthew N. Murray, and Beth Glenn. 2019. "Prescription Opioids and Labor Market Pains: The Effect of Schedule II Opioids on Labor Force Participation and Unemployment." Journal of Human Resources. doi: 10.3368/jhr.55.4.0517-8782R1.

Morden, Nancy E., Jeffrey C. Munson, Carrie H. Colla, Jonathan S. Skinner, Julie P.W. Bynum, Weiping Zhou, and Ellen R. Meara. 2014. "Prescription Opioid Use Among Disabled Medicare Beneficiaries: Intensity, Trends, and Regional Variation." Medical Care 52: 852-859.

Mytelka, Christine M., Melanie K. Kuester, Colin R. Gray, and Lucas Everheart. 2018. "How Changing Opioid-Prescribing Patterns Can Impact Risk Scores: Medicaid Expansion CDPS + Rx Analysis.” Seattle, WA: Milliman.

Scherrer, Jeffrey F., Joanne Salas, Laurel A. Copeland, Eileen M. Stock, Brian K. Ahmedani, Mark D. Sullivan, Thomas Burroughs, David Schneider, Kathleen K. Bucholz, and Patrick J. Lustman. 2016. "Prescription Opioid Duration, Dose, and Increased Risk of Depression in Three Large Patient Populations." Annals of Family Medicine 14(1): pp. 54-62.

Schiltenwolf, M., M. Akbar, A. Hug, U. Pfuller, S. Gantz, E. Neubauer, H. Flor, and H. Wang. 2014. "Evidence of Specific Cognitive Deficits in Patients with Chronic Low Back Pain Under Long-Term Substitution Treatment of Opioids.” Pain Physician 17(1): 9-20.

Serdarevic, Mirsada, Catherine W. Striley, and Linda B. Cottler. 2017. "Gender Differences in Prescription Opioid Use." Current Opinion in Psychiatry 30(4): 238-246.

U.S. Department of Health and Human Services. "What Is the U.S. Opioid Epidemic?" September 4, 2019 (last reviewed). Available at https://www.hhs.gov/opioids/about-theepidemic/index.html. Accessed January 21, 2020.

U.S. Social Security Administration. 2018. "Monthly Statistical Snapshot, October 2018." Washington, DC.

U.S. Social Security Administration. 2019. "Annual Statistical Supplement to the Social Security Bulletin, 2018.” SSA Publication No. 13-11700. Washington, DC. 
Vowles, Kevin E., Mindy L. McEntee, Peter Siyahhana Julnes, Tessaa Frohe, John P. Ney, and David N. van der Goes. 2015. "Rates of Opioid Misuse, Abuse, and Addiction in Chronic Pain: A Systematic Review and Data Synthesis." Pain 156(4): 569-576. doi: 10.1097/01.j.pain.0000460357.01998.f1.

Wu, April Yanyuan, Peter Mariani, Jia Pu, and Andrew Hurwitz. 2019. "New Statistics on Opioid Use Among SSDI Applicants and Their Policy Implications." DRC Working Paper. Chicago, IL: Mathematica. 


\section{Exhibit 1. Mode of Medication Reporting}

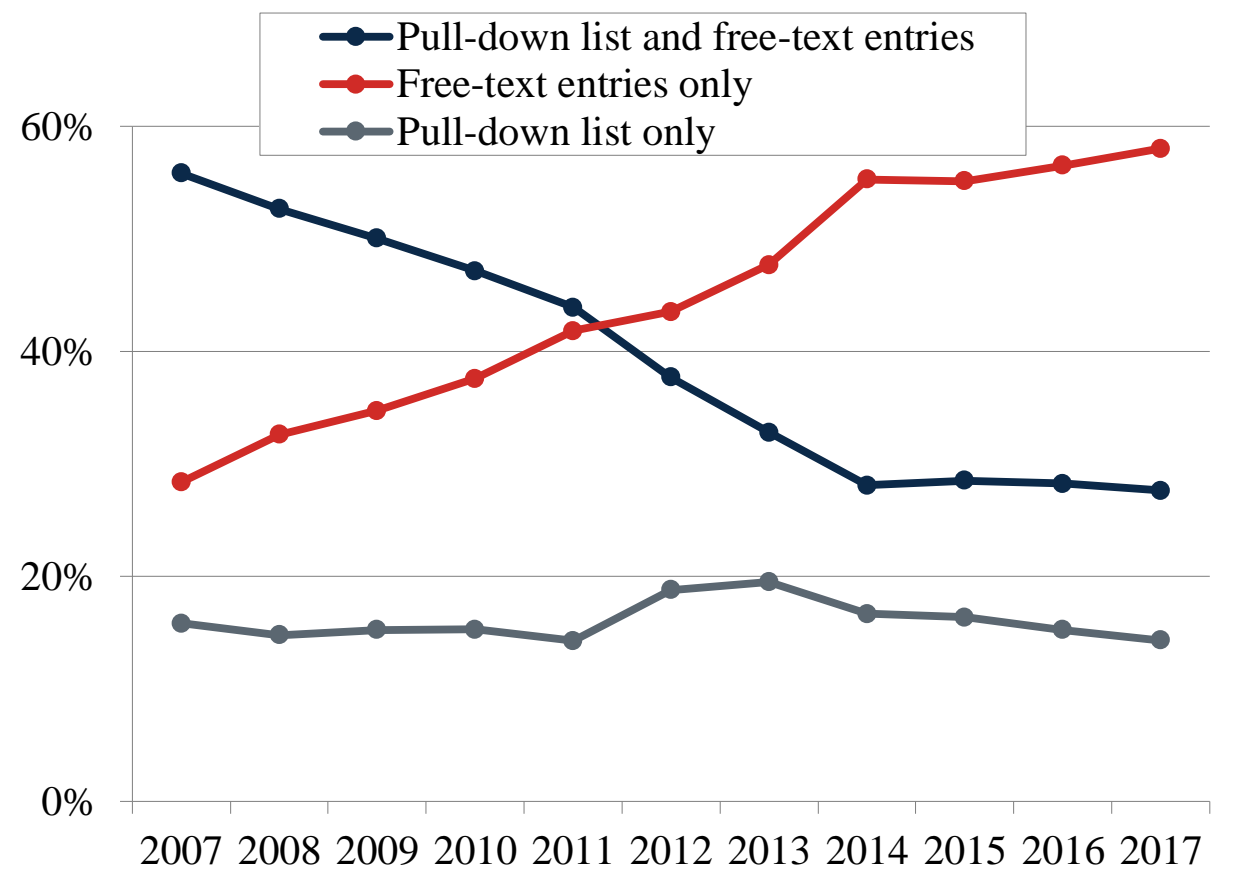

Source: Authors' calculations based on the 2007-2017 SDR.

Exhibit 2. Prevalence of Opioid Use among Applicants

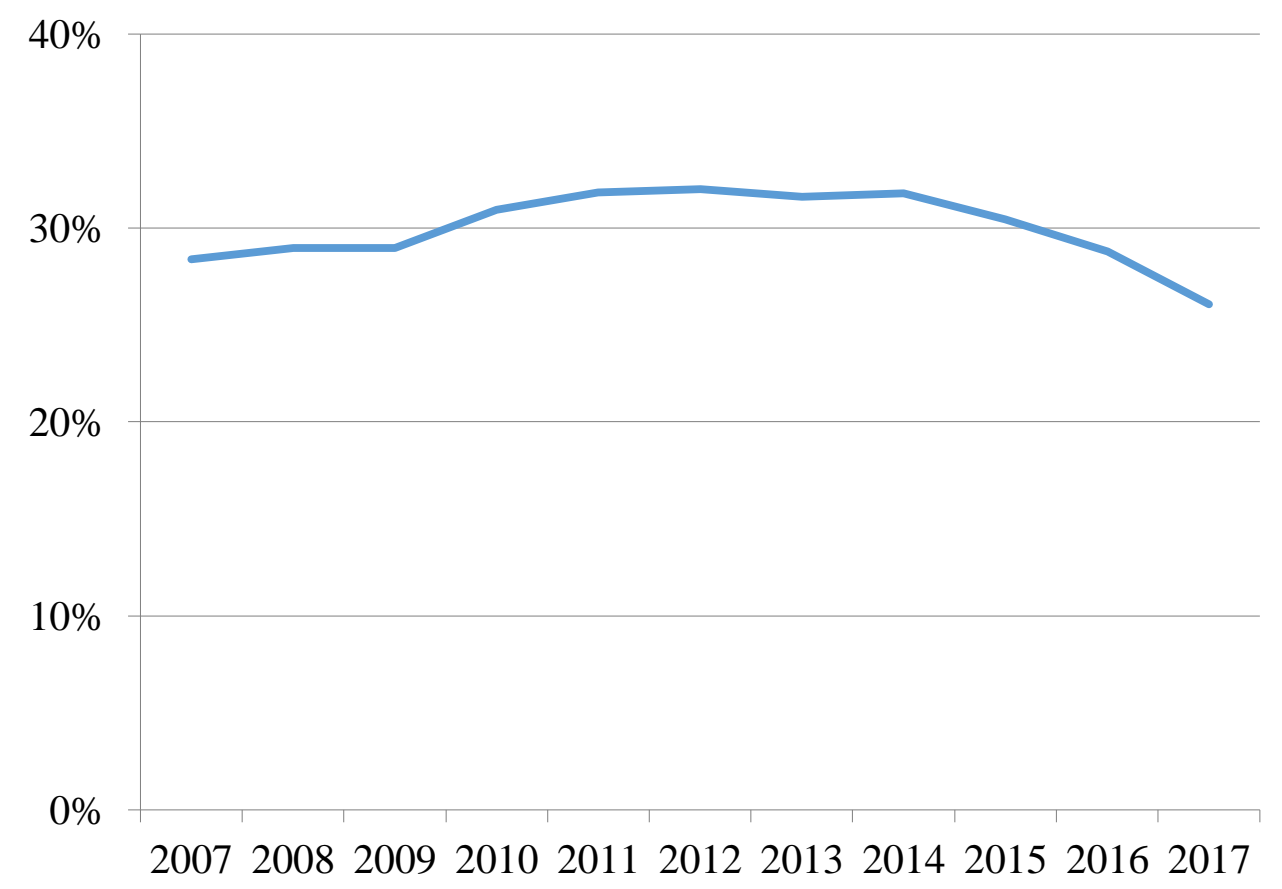

Source: Authors' calculations based on the 2007-2017 SDR. 
Exhibit 3. Prevalence of Opioid Use by Applicant Characteristics

a. Prevalence of Opioid Use by Application Type

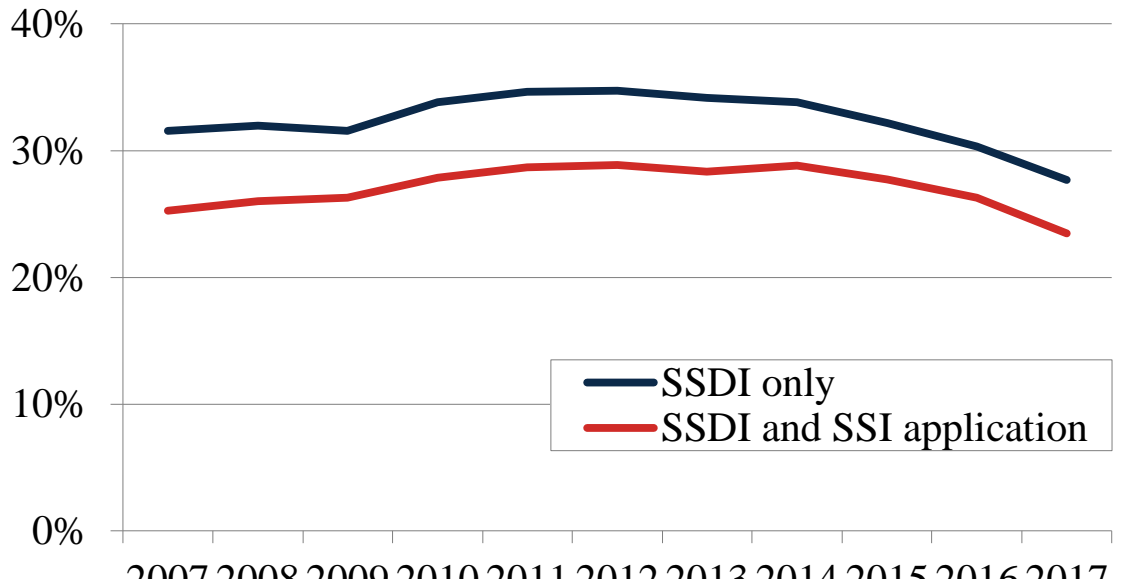

20072008200920102011201220132014201520162017

c. Prevalence of Opioid Use by Education (Age 22+)

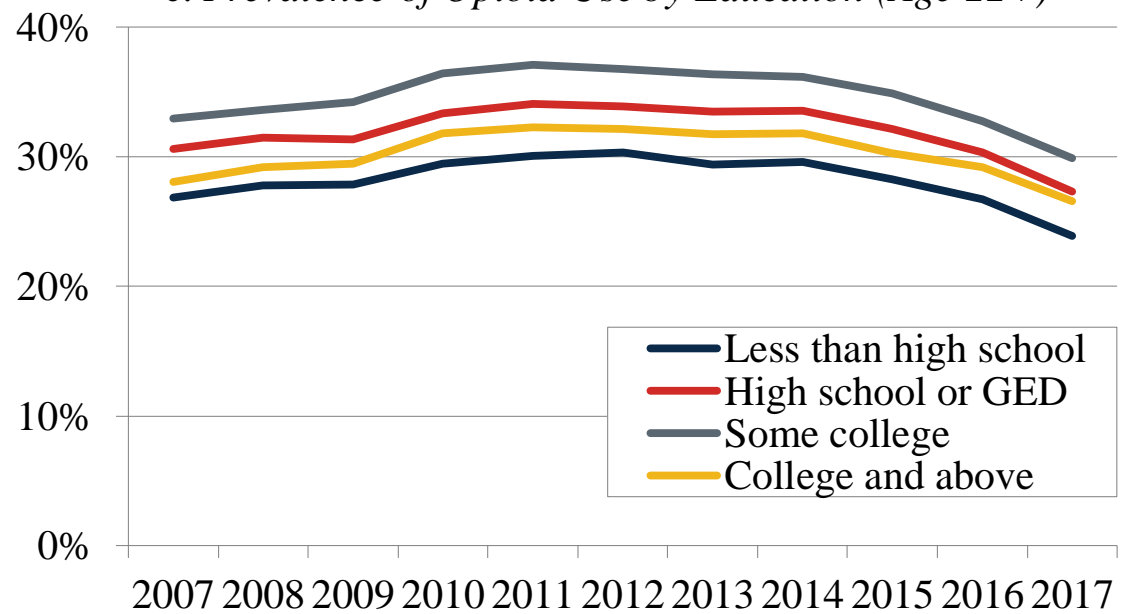

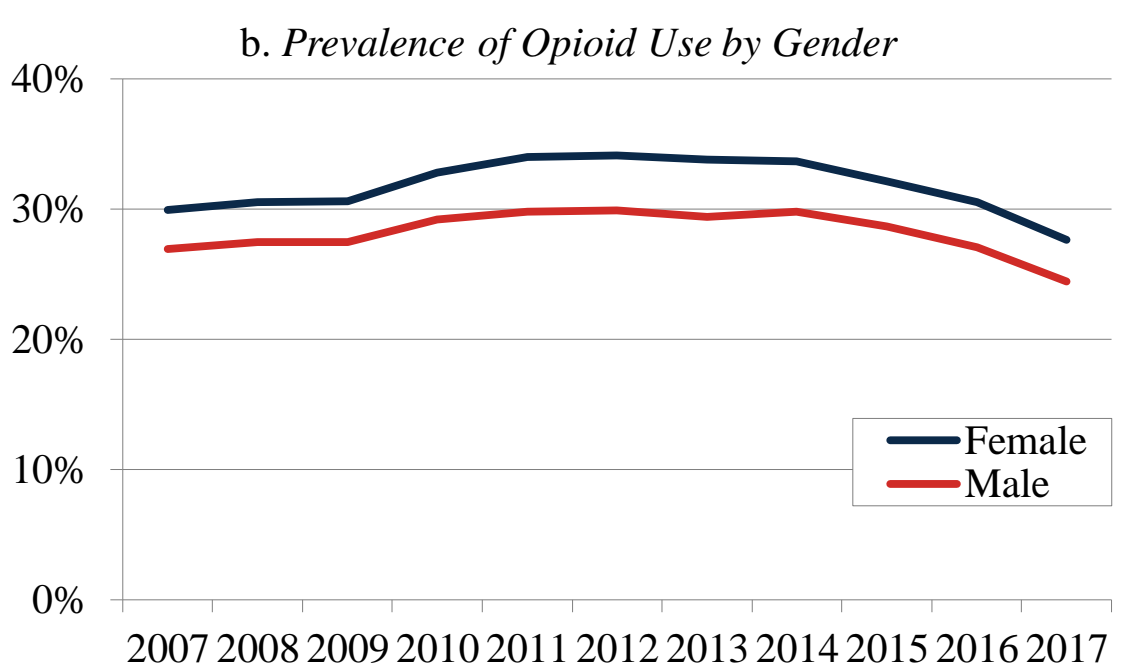

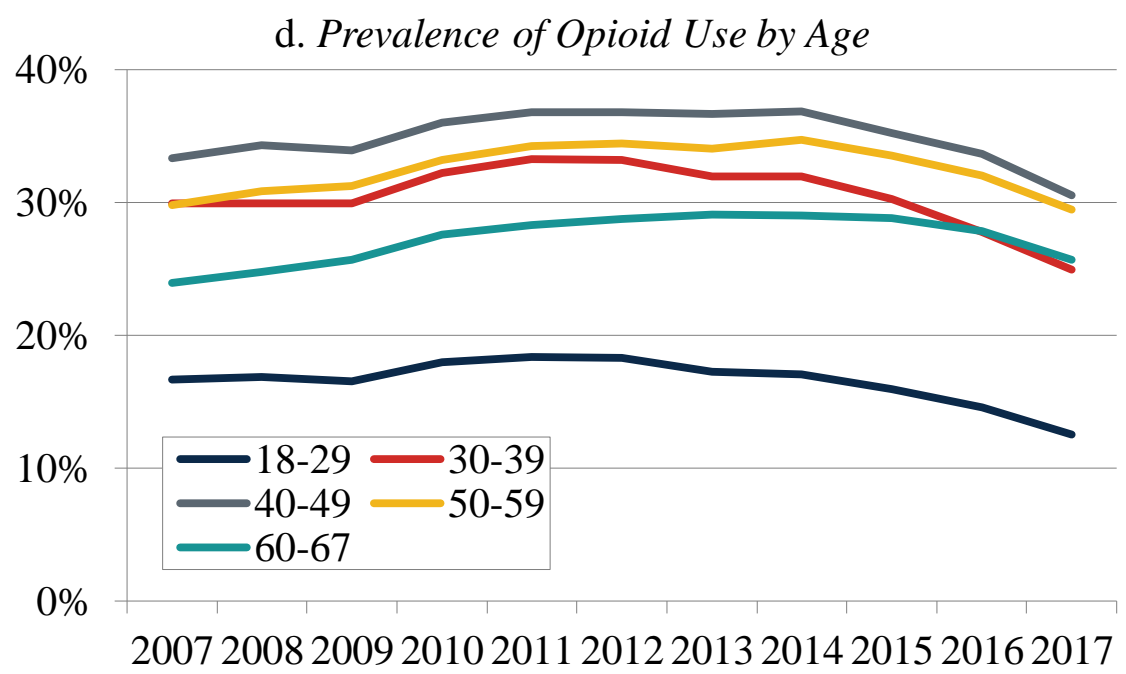

Notes: In each year of the analysis period, the difference between demographic subgroups was statistically significant at the 1 percent level. We tested statistical significance by using a t-test for gender and application type (SSDI-only or SSDI and SSI) and by using a chi-square test for age and education.

Source: Authors' calculations based on the 2007-2017 SDR. 

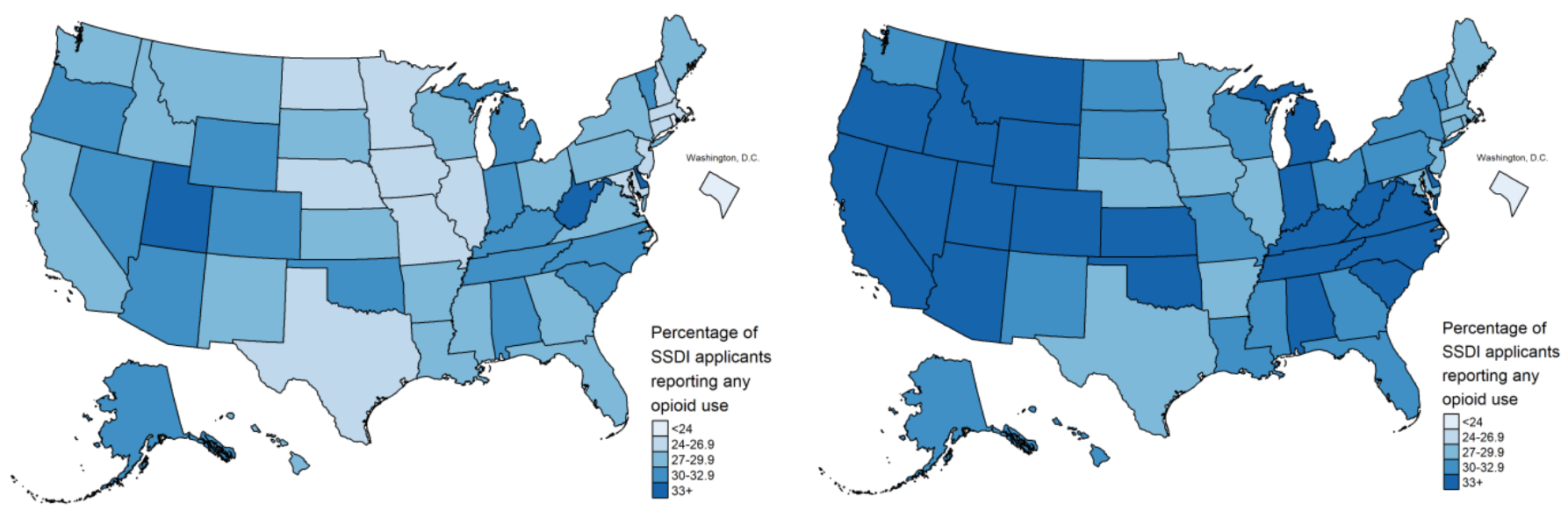

2017

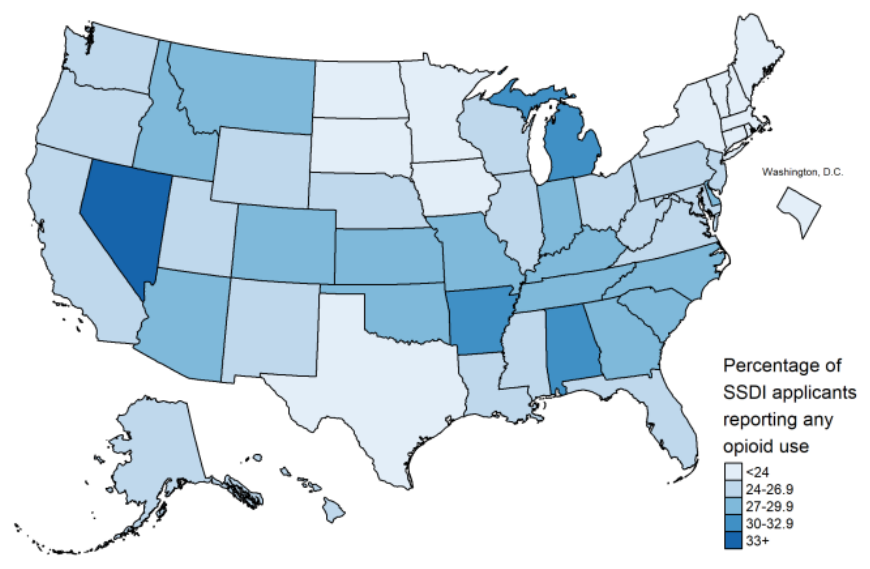

Note: The full statistics by state are available upon request. Source: Authors' calculations based on the 2007-2017 SDR. 
Exhibit 5. Association Between Applicant Opioid Use in 2009 and Post-Application Outcomes in 2014

\begin{tabular}{lcc} 
2014 outcome & $\begin{array}{c}\text { Unadjusted rate of outcome } \\
\text { for applicants without } \\
\text { reported opioid use } \\
\text { (percent) }\end{array}$ & $\begin{array}{c}\text { Average marginal effect } \\
\text { of opioid use } \\
\text { (percentage point) }\end{array}$ \\
\hline Awarded SSDI benefits and alive & $51.6 \%$ & $4.1 \% * * *(0.1)$ \\
Awarded SSDI benefits and dead & 5.7 & $1.5^{* * *}(0.1)$ \\
Not awarded SSDI benefits and alive & 39.6 & $-6.5^{* * *}(0.1)$ \\
Not awarded SSDI benefits and dead & 3.2 & $0.8^{* * *}(0.1)$ \\
\hline
\end{tabular}

Notes: Delta-method standard errors are in parentheses. ${ }^{* * *}$ indicates statistical significance at the 1 percent level. 


\section{RECENT WORKING PAPERS FROM THE CENTER For RETIREMENT RESEARCH AT BOSTON COLLEGE}

The Value of Annuities

Gal Wettstein, Alicia H. Munnell, Wenliang Hou, and Nilufer Gok, March 2021

Will Women Catch Up to Their Fertility Expectations?

Anqi Chen and Nilufer Gok, February 2021

The Consequences of Current Benefit Adjustments for Early and Delayed Claiming

Andrew G. Biggs, Anqi Chen, and Alicia H. Munnell, January 2021

Intended Bequests and Housing Equity in Older Age

Gary V. Engelhardt and Michael D. Eriksen, January 2021

The Effect of Early Claiming Benefit Reduction on Retirement Rates

Damir Cosic and C. Eugene Steuerle, January 2021

Financial Security at Older Ages

Barbara A. Butrica and Stipica Mudrazija, December 2020

Do People Work Longer When They Live Longer?

Damir Cosic, Aaron R. Williams, and C. Eugene Steuerle, December 2020

Do State and Local Government Employees Save Outside of Their Defined Benefit Plans When They Need To?

Laura D. Quinby and Geoffrey T. Sanzenbacher, November 2020

How Much Taxes Will Retirees Owe on Their Retirement Income?

Anqi Chen and Alicia H. Munnell, November 2020

A Behavioral Economics Assessment of SSDI Earnings Reporting Documents

Denise Hoffman, Jonah Deutsch, and Britta Seifert, November 2020

How Accurate Are Retirees' Assessments of Their Retirement Risk?

Wenliang Hou, July 2020

Is Nontraditional Work at Older Ages Associated with Better Retirement Security? Matthew S. Rutledge and Gal Wettstein, July 2020

New Insights on Self-Employment of Older Adults in the United States Joelle Abramowitz, July 2020

All working papers are available on the Center for Retirement Research website (https://crr.bc.edu) and can be requested by e-mail (crr@bc.edu) or phone (617-552-1762). 